\title{
A PROPERTY OF THE ZEROS OF LEGENDRE POLYNOMIALS
}

\author{
BY \\ A. ZYGMUND
}

$\$ 1$

1. Let $m$ and $n$ be any two positive integers such that

$$
m / n \geqq 1+\delta,
$$

where $\delta$ is positive. It has been proved that for any trigonometric polynomial

$$
T(\theta)=(1 / 2) a_{0}+\sum_{\nu=1}^{n}\left(a_{\nu} \cos \nu \theta+b_{\nu} \sin \nu \theta\right)
$$

of $\operatorname{order} n$,

$$
\max _{0 \leqq \theta \leqq 2 \pi}|T(\theta)| \leqq A_{\delta}^{\prime} \max _{\mu=0,1, \cdots, 2 m}\left|T\left(\frac{2 \pi \mu}{2 m+1}\right)\right|,
$$

where the coefficient $A_{\delta}^{\prime}$ depends on $\delta$ only ${ }^{1}{ }^{1}$.

Similarly, for $r \geqq 1$

$$
\left\{\frac{1}{2 \pi} \int_{0}^{2 \pi}|T(\theta)| r d \theta\right\}^{3 / r} \leqq B_{\delta}^{\prime} \frac{1}{2 m+1}\left\{\sum_{\mu=0}^{2 m}\left|T\left(\frac{2 \pi \mu}{2 m+1}\right)\right|^{r}\right\}^{1 / r}
$$

with $B_{\delta}^{\prime}$ depending on $\delta$ only.

It is the main purpose of this note to show that there exist analogous results for ordinary polynomials

$$
Q(x)=c_{0}+c_{1} x+\cdots+c_{n} x^{n},
$$

if instead of the points $2 \pi \mu /(2 m+1)$ we consider the zeros of the Legendre polynomial $P_{m}(x)$, where $m$ satisfies the inequality (1.1). These zeros will be denoted by $(2)$

Presented to the Society, December 27, 1942; received by the editors December 1, 1942.

(1) See S. Bernstein, Sur une classe de formules d'interpolation, Bull. Acad. Sci. URSS. (7) vol. 4 (1931) pp. 1151-1161. A proof of (1.3) (as well as that of inequality (1.4) below) was also given by J. Marcinkiewicz and A. Zygmund, Mean values of trigonometric polynomials, Fund. Math. vol. 28 (1937) pp. 131-166, especially p. 148. The authors overlooked the earlier paper of Bernstein.

(2) Of course, the inequalities (1.3) and (1.4) for trigonometric polynomials may be easily translated into analogous results for ordinary polynomials, if we take for the $x_{\nu}^{(m)}$ the zeros of Tchebyscheff's polynomials. The case of Legendre abscissas requires, however, an entirely different argument. 


$$
x_{1}^{(m)}, x_{2}^{(m)}, \cdots, x_{m}^{(m)} .
$$

By $\gamma_{1}^{(m)}, \gamma_{2}^{(m)}, \cdots, \gamma_{m}^{(m)}$ we shall denote the Christoffel numbers, that is the numbers such that

$$
\int_{-1}^{+1} R(x) d x=\sum_{\mu=1}^{m} R\left(x_{\mu}^{(m)}\right) \gamma_{\mu}^{(m)},
$$

for any polynomial $R(x)$ of degree not exceeding $2 m-1$. It is very well known that these numbers are positive $\left.{ }^{3}\right)$.

Theorem 1. Suppose that $m$ and $n$ are positive integers, $n<m$, and that $r$ is any real number not less than 1 . Then for any polynomial $Q(x)$ of degree $n$,

$$
\begin{gathered}
\max _{-1 \leqq x \leqq+1}|Q(x)| \leqq K_{m, n} \max _{1 \leqq \mu \leqq m}\left|Q\left(x_{\mu}^{(m)}\right)\right|, \\
\left\{\int_{-1}^{+1}|Q(x)|^{r} d x\right\}^{1 / r} \leqq K_{m, n}\left\{\sum_{\mu=1}^{m}\left|Q\left(x_{\mu}^{(m)}\right)\right|^{r} \gamma_{\mu}^{(m)}\right\}^{1 / r},
\end{gathered}
$$

where $K_{m, n}$ depends on $m$ and $n$ only. If $m$ and $n$ satisfy (1.1) then

$$
K_{m, n} \leqq A_{\delta}
$$

with $A_{\delta}$ depending only on $\delta$.

2. The proof of the inequalities (1.8) and (1.9) has certain features in common with that of (1.3) and (1.4). In the latter case, the main idea of the proof was that to a partial sum of a trigonometric Fourier series we may add a nonoverlapping group of terms so that the resulting expression is a simple linear combination of the first arithmetic means of the Fourier series. In the proof of (1.8) and (1.9) we shall apply a similar device to Fourier-Legendre series, but instead of the first we shall consider the second arithmetic means of the series.

Let us consider an arbitrary series $u_{0}+u_{1}+\cdots+u_{n}+\cdots$. The partial sums and the second Cesàro means of the series we shall denote by $s_{n}$ and $\sigma_{n}^{\prime \prime}$, respectively. It is easy to see that, given three non-negative integers $n<p<q$, we can always find constants $A, B, C$ (depending on $n, p, q$ only) such that

$$
A \sigma_{n}^{\prime \prime}+B \sigma_{p}^{\prime \prime}+C \sigma_{q}^{\prime \prime}-s_{n+2}
$$

contains only terms $u_{\nu}$ with $\nu>n+2$.

For the proof of this remark, we observe that

(3) See, for example, G. Szegö, Orthogonal polynomials, Amer. Math. Soc. Colloquium Publications vol. 23 (1939) p. $46 \mathrm{ff}$. 


$$
\sigma_{n}^{\prime \prime}=\sum_{\nu=0}^{n}\left(1-\frac{\nu}{n+1}\right)\left(1-\frac{\nu}{n+2}\right) u_{\nu}=\sum_{\nu=0}^{n+2}\left(1-\frac{\nu}{n+1}\right)\left(1-\frac{\nu}{n+2}\right) u_{\nu}
$$

and that similar formulas hold for $\sigma_{p}^{\prime \prime}$ and $\sigma_{q}^{\prime \prime}$. This gives for the determination of the numbers $A, B, C$ the equations

$$
\begin{gathered}
A+B+C=1, \\
A\left(\frac{1}{n+1}+\frac{1}{n+2}\right)+B\left(\frac{1}{p+1}+\frac{1}{p+2}\right)+C\left(\frac{1}{q+1}+\frac{1}{q+2}\right)=0, \\
\frac{A}{(n+1)(n+2)}+\frac{B}{(p+1)(p+2)}+\frac{C}{(q+1)(q+2)}=0 .
\end{gathered}
$$

Hence

(2.2) $A=\frac{(n+1)(n+2)}{(p-n)(q-n)}, C=\frac{(q+1)(q+2)}{(q-p)(q-n)}, B=-\frac{(p+1)(p+2)}{(q-p)(p-n)}\left({ }^{4}\right)$.

The next remark we need is the following theorem of Fejér $\left(^{5}\right):$ The second Cesàro means of the series

$$
\sum_{\nu=0}^{\infty}(\nu+1 / 2) P_{\nu}(x) P_{\nu}(t)
$$

are non-negative for $-1 \leqq x \leqq+1,-1 \leqq t \leqq+1$.

Let us denote these means by $K_{n}^{\prime \prime}(x, t)$ and let $\sigma_{n}^{\prime \prime}(x ; f)$ denote the second Cesàro means of the Fourier-Legendre series

$$
\sum_{\nu=0}^{\infty} a_{\nu} P_{\nu}(x)
$$

of a function $f(x)$ defined in the interval $-1 \leqq x \leqq+1$. Then

$$
\sigma_{n}^{\prime \prime}(x ; f)=\int_{-1}^{+1} f(t) K_{n}^{\prime}(x, t) d t .
$$

Hence

$$
\begin{aligned}
\max _{-1 \leqq x \leqq+1}\left|\sigma_{n}^{\prime}(x ; f)\right| & \leqq \max _{-1 \leqq t \leqq+1}|f(t)| \cdot \int_{-1}^{+1} K_{n}^{\prime \prime}(x, t) d t \\
& =\max _{-1 \leqq x \leqq+1}|f(x)| .
\end{aligned}
$$

(4) Thus the numbers $A,-B, C$ are positive.

(5) L. Fejér, Über die Laplacesche Reihe, Math. Ann. vol. 67 (1909) pp. 76-109. 
Again, if $r \geqq 1$ and $1 / r+1 / r^{\prime}=1$, then

so that

$$
\begin{aligned}
\left|\sigma_{n}^{\prime \prime}(x ; f)\right| & \leqq \int_{-1}^{+1}|f(t)| K_{n}^{\prime \prime 1 / r} K_{n}^{1 / r^{\prime}} d t \\
& \leqq\left\{\int_{-1}^{+1}|f(t)|^{r} K_{n}^{\prime \prime}(x, t) d t\right\}^{1 / r}\left\{\int_{-1}^{+1} K_{n}^{\prime \prime}(x, t) d t\right\}^{1 / r^{\prime}},
\end{aligned}
$$

$$
\left|\sigma_{n}^{\prime \prime}(x ; f)\right|^{r} \leqq \int_{-1}^{+1}|f(t)|^{r} K_{n}^{\prime}(x, t) d t .
$$

Integrating both sides of the inequality over the interval $-1 \leqq x \leqq+1$, and inverting the order of integration on the right, we get

$$
\int_{-1}^{+1}\left|\sigma_{n}^{\prime \prime}(x ; f)\right| r d x \leqq \int_{-1}^{+1}|f(t)|^{r} d t .
$$

3. We shall now prove the following theorem which is in a sense a converse of the inequality (1.9), and which gives slightly more than we actually need for the proof of Theorem 1.

LemMA. Let $r \geqq 1$ and let $m$ and $n$ be two positive integers such that $n \leqq 2 m-1$. Then, for any polynomial $R(x)$ of degree $n$,

$$
\left\{\sum_{\nu=1}^{m}\left|R\left(x_{\nu}^{(m)}\right)\right|^{r} \gamma_{\nu}^{(m)}\right\}^{1 / r} \leqq L_{m, n}\left\{\int_{-1}^{+1}|R(x)|^{r} d x\right\}^{1 / r},
$$

where the coefficient $L_{m, n}$ depends on $m$ and $n$ only. If

$$
n(1+\delta) \leqq 2 m-1 \text {, }
$$

then

$$
L_{m, n} \leqq L\left(1+1 / \delta^{2}\right),
$$

where $L$ is an absolute constant.

Proof. Let $\mu$ be any integer such that $n-2<\mu<2 m-1$, and let $A^{*}, B^{*}, C^{*}$ denote the numbers defined by the formulas (2.2) with $n, p, q$ replaced by $n-2, \mu, 2 m-1$, respectively $\left(^{6}\right)$. Hence, treating $R(x)$ as a Fourier-Legendre series, we get

$$
R(x)=A^{*} \sigma_{n-2}^{\prime \prime}(x ; R)+B^{*} \sigma_{\mu}^{\prime \prime}(x ; R)+C^{*} \sigma_{2 m-1}^{\prime \prime}(x ; R) .
$$

Now let $\lambda$ be any positive integer not exceeding $2 m-1$, and let us consider the inequality (2.6) with $f$ replaced by $R$ and $n$ by $\lambda$. We get

(6) The argument is valid even if $n=0$ or $n=1$, provided we set $\sigma_{-1}^{\prime \prime}=\sigma_{-2}^{\prime \prime}=0$. 


$$
\begin{aligned}
\sum_{\nu=1}^{m}\left|\sigma_{\lambda}{ }^{\prime \prime}\left(x_{\nu}{ }^{(m)} ; R\right)\right|^{r} \gamma_{\nu}^{(m)} & \leqq \int_{-1}^{+1}|R(t)|^{r}\left\{\sum_{\nu=1}^{m} \gamma_{\nu}^{(m)} K_{\lambda}{ }^{\prime \prime}\left(x_{\nu}{ }^{(m)}, t\right)\right\} d t \\
& =\int_{-1}^{+1}|R(t)|^{r} d t,
\end{aligned}
$$

since

$$
\sum_{\nu=1}^{m}{\gamma_{\nu}}_{\nu}^{(m)} K_{\lambda}^{\prime \prime}\left(x_{\nu}^{(m)}, t\right)=\int_{-1}^{+1} K_{\lambda}^{\prime \prime}(x, t) d t=1 .
$$

Hence, on account of (3.4) and using Minkowski's inequality,

This proves (3.1) with

$$
\begin{aligned}
\left\{\sum_{\nu=1}^{m}\left|R\left(x_{\nu}^{(m)}\right)\right|^{r} \gamma_{\nu}^{(m)}\right\}^{1 / r} \leqq & A^{*}\left\{\sum_{\nu=1}^{m}\left|\sigma_{n-2}^{\prime \prime}\left(x_{\nu}^{(m)} ; R\right)\right|^{r} \gamma_{\nu}^{(m)}\right\}^{1 / r} \\
& +\left|B^{*}\right|\left\{\sum_{\nu=1}^{m}\left|\sigma_{\mu}^{\prime \prime}\left(x_{\nu}^{(m)} ; R\right)\right|^{r} \gamma_{\nu}^{(m)}\right\}^{1 / r} \\
& +C^{*}\left\{\sum_{\nu=1}^{m} \sigma_{2 m-1}^{\prime \prime}\left(x_{\nu}^{(m)} ; R\right)^{r} \gamma_{\nu}^{(m)}\right\}^{1 / r} \\
\leqq & \left(A^{*}+\left\{B^{*} \mid+C^{*}\right)\left\{\int_{-1}^{+1}|R(t)|^{r} d t\right\}^{1 / r} .\right.
\end{aligned}
$$

$$
L_{m, n}=A^{*}+\left|B^{*}\right|+C^{*} .
$$

In order to prove (3.3) we assume that we have equality in (3.2), for this only increases $\delta$, and we set

$$
\mu=[((n-2)+(2 m-1)) / 2]=m-1+[(n-1) / 2] .
$$

Then

$$
\begin{gathered}
2 m-1-(n-2)>2 m-1-n=n \delta \\
2 m-1-\mu=m-[(n-1) / 2] \geqq m-(1 / 2)(n-1) \\
>(1 / 2)(2 m-1-n)=(1 / 2) n \delta, \\
\mu-(n-2)=m+[(n-1) / 2]-(n-1) \geqq m-(1 / 2)(n-1)-1 \\
=(1 / 2)(2 m-1-n)=(1 / 2) n \delta .
\end{gathered}
$$

Hence the denominators in (2.2), with $n, p, q$ replaced by $n-2, \mu, 2 m-1$, respectively, all exceed $(1 / 4) n^{2} \delta^{2}=(1 / 4)(2 m-1)^{2} \delta^{2}(1+\delta)^{-2}$. The numerators are $O\left(m^{2}\right)$. From this and (3.6) we deduce (3.3).

4. We now pass to the proof of (1.9). We note that

$$
\left\{\int_{-1}^{+1}|Q(x)| r d x\right\}^{1 / r}=\max _{f} \int_{-1}^{+1} Q(x) f(x) d x,
$$


for all $f$ with

$$
\left\{\int_{-1}^{+1}|f| r^{\prime} d x\right\}^{1 / r^{\prime}} \leqq 1 .
$$

For $f$ we may take continuous functions only, or even only polynomials. We now observe that, if $s_{n}(x ; f)$ denotes the $n$th partial sum of the FourierLegendre series of $f(x)$, then

$$
\int_{-1}^{+1} Q(x) f(x) d x=\int_{-1}^{+1} Q(x) s_{n}(x ; f) d x=\int_{-1}^{+1} Q(x) T(x) d x,
$$

where $T(x)$ is any polynomial such that the $n$th partial sum of its FourierLegendre development coincides with $s_{n}(x ; f)$. Let us set

$$
T(x)=A^{\prime} \sigma_{n-2}^{\prime \prime}(x ; f)+B^{\prime} \sigma_{p}^{\prime \prime}(x ; f)+C^{\prime} \sigma_{m}^{\prime \prime}(x ; f),
$$

where $p$ is any integer satisfying $n-2<p<m$, and the numbers $A^{\prime}, B^{\prime}, C^{\prime}$ are defined by (2.2) with $n, p, q$ replaced by $n-2, p, m$, respectively. Since $Q(x) T(x)$ is of degree $n+m \leqq 2 m-1$,

$$
\begin{aligned}
\int_{-1}^{+1} Q(x) T(x) d x & =\sum_{\nu=1}^{m} Q\left(x_{\nu}{ }^{(m)}\right) T\left(x_{\nu}{ }^{(m)}\right) \gamma_{\nu}{ }^{(m)} \\
& \leqq\left\{\sum_{\nu=1}^{m}\left|Q\left(x_{\nu}{ }^{(m)}\right)\right|^{r} \gamma_{\nu}{ }^{(m)}\right\}^{1 / r}\left\{\sum_{\nu=1}^{m}\left|T\left(x_{\nu}{ }^{(m)}\right)\right|^{\left.r^{\prime} \gamma_{\nu}{ }^{(m)}\right\}^{1 / r^{\prime}} .}\right.
\end{aligned}
$$

Applying Lemma 1 to the last sum and denoting by $L^{\prime}$ the least upper bound of the sequence $L_{m, m}\left(L^{\prime}\right.$ is finite, on account of (3.3)), we get

$$
\begin{aligned}
\left\{\sum_{\nu=1}^{m}\left|T\left(x_{\nu}^{(m)}\right)\right|^{r^{\prime}} \gamma_{\nu}^{(m)}\right\}^{1 / r^{\prime}} & \leqq L_{m, m}\left\{\int_{-1}^{+1}|T(x)|^{r^{\prime}} d x\right\}^{1 / r^{\prime}} \leqq L^{\prime}\left\{\int_{-1}^{+1}|T(x)|^{r^{\prime}} d x\right\}^{1 / r^{\prime}} \\
\leqq & L^{\prime}\left[A^{\prime}\left\{\int_{-1}^{+1}\left|\sigma_{n-2}^{\prime \prime}\right|^{r^{\prime}} d x\right\}^{1 / r^{\prime}}+\left|B^{\prime}\right|\left\{\int_{-1}^{+1}\left|\sigma_{p}^{\prime \prime}\right|^{r^{\prime}} d x\right\}^{1 / r^{\prime}}\right. \\
& \left.+C^{\prime}\left\{\int_{-1}^{+1}\left|\sigma_{m}^{\prime \prime}\right|^{r^{\prime}} d x\right\}^{1 / r^{\prime}}\right] \\
\leqq & L^{\prime}\left(A^{\prime}+\left|B^{\prime}\right|+C^{\prime}\right)\left\{\int_{-1}^{+1}|f|^{r^{\prime}} d x\right\}^{1 / r^{\prime}} \leqq K_{m, n},
\end{aligned}
$$

with 


$$
K_{m, n}=L^{\prime}\left(A^{\prime}+\left|B^{\prime}\right|+C^{\prime}\right) .
$$

From this, (4.1), (4.3), and (4.5) we deduce (1.9). It may be added that, if $r=1$, the above argument does not require Lemma 1 . What it uses is the trivial inequality.

$$
\max _{\nu=1, \cdots, m}\left|Q\left(x_{\nu}^{(m)}\right)\right| \leqq \max _{-1 \leqq x \leqq+1}|Q(x)|
$$

(which of course is contained in (3.1) for $r=+\infty$ ).

The proof of the inequality (1.8) is essentially the same as that of (1.9). Instead of (4.1) and (4.2) we use the relation

$$
\max _{-1 \leqq x \leqq+1}|Q(x)|=\max _{f} \int_{-1}^{+1} Q(x) f(x) d x, \text { for } \int_{-1}^{+1}|f| d x \leqq 1 .
$$

The rest of the argument is unchanged.

5. In order to prove (1.10), we take

$$
p=[((n-2)+m) / 2]=[(n+m) / 2]-1
$$

Then (the argument is analogous to (3.7))

$$
\begin{aligned}
& m-(n-2)>m-n \geqq n \delta, \\
m-p & =m-[(n+m) / 2]+1>m-(n+m) / 2 \\
& =(1 / 2)(m-n) \geqq(1 / 2) n \delta, \\
p-(n-2)= & {[(m+n) / 2]+1-n>(m+n) / 2-n } \\
= & (1 / 2)(m-n) \geqq(1 / 2) n \delta,
\end{aligned}
$$

so that the denominators of $A^{\prime}, B^{\prime}, C^{\prime}$ are not less than $(1 / 4) n^{2} \delta^{2}=(1 / 4) m^{2}$ $\cdot(1+\delta)^{-2} \delta^{2}$, assuming, as we may, that $m=n(1+\delta)$. Since the numerators are $O\left(m^{2}\right)$, we see that, assuming (1.1)

$$
K_{m, n} \leqq K\left(1+\delta^{-2}\right),
$$

where $K$ is an absolute constant. This concludes the proof of Theorem 1.

$\S 2$

6. The argument used in $\$ 1$ may be applied to some other problems. As an example, we shall prove the following:

THEOREM 2. Let $r \geqq 1$ and let $m$ and $n$ be any two positive integers such that $m \geqq n$. Then every polynomial $R(x)$ of degree $n$ may be represented in the form

$$
R(x)=R_{1}(x)-R_{2}(x),
$$

where $R_{1}$ and $R_{2}$ are polynomials of degrees at most $m$, are non-negative for 
$-1 \leqq x \leqq+1$, and satisfy the inequalities

$$
\left\{\int_{-1}^{+1} R_{i}^{r}(x) d x\right\}^{1 / r} \leqq K_{m, n}\left\{\int_{-1}^{+1}|R(x)|^{r} d x\right\}^{1 / r}
$$

with $K_{m, n}$ depending on $m$ and $n$ only. If $m$ and $n$ satisfy (1.1), then

$$
K_{m, n} \leqq K\left(1+\delta^{-2}\right)
$$

where $K$ is an absolute constant.

Proof. Let us set

$$
R(x)=R^{+}(x)-R^{-}(x)
$$

where

$$
R^{+}(x)=\max \{R(x), 0\}, R^{-}(x)=\max \{-R(x), 0\}
$$

so that

$$
\begin{gathered}
0 \leqq R^{+}(x) \leqq|R(x)|, \quad 0 \leqq R^{-}(x) \leqq|R(x)| \\
\int_{-1}^{+1}\left\{R^{+}(x)\right\} r d x \leqq \int_{-1}^{+1}|R(x)|^{r} d x, \quad \int_{-1}^{+1}\left\{R^{-}(x)\right\} r d x \leqq \int_{-1}^{+1}|R(x)|^{r} d x .
\end{gathered}
$$

Let $p$ be any in teger satisfying the inequalities

$$
n-2<p<m
$$

and let $A^{\prime}, B^{\prime}, C^{\prime}$ be defined by the formulas (2.2) with $n, p, q$ replaced by $n-2, p, m$, respectively. Let us consider the expressions

$$
\begin{aligned}
& T^{*}(x)=A^{\prime} \sigma_{n-2}^{\prime \prime}\left(x ; R^{+}\right)+B^{\prime} \sigma_{p}^{\prime \prime}\left(x ; R^{+}\right)+C^{\prime} \sigma_{m}^{\prime \prime}\left(x ; R^{+}\right), \\
& T_{*}(x)=A^{\prime} \sigma_{n-2}^{\prime \prime}\left(x ; R^{-}\right)+B^{\prime} \sigma_{p}^{\prime \prime}\left(x ; R^{-}\right)+C^{\prime} \sigma_{m}^{\prime \prime}\left(x ; R^{-}\right)
\end{aligned}
$$

whose difference is

$$
A^{\prime} \sigma_{n-2}^{\prime \prime}(x ; R)+B^{\prime} \sigma_{p}^{\prime \prime}(x ; R)+C^{\prime} \sigma_{m}^{\prime \prime}(x ; R)=R(x),
$$

and let us set

$$
\begin{aligned}
& R_{1}(x)=A^{\prime} \sigma_{n-2}^{\prime \prime}\left(x ; R^{+}\right)-B^{\prime} \sigma_{p}^{\prime \prime}\left(x ; R^{-}\right)+C^{\prime} \sigma_{m}^{\prime \prime}\left(x ; R^{+}\right), \\
& R_{2}(x)=A^{\prime} \sigma_{n-2}^{\prime \prime}\left(x ; R^{-}\right)-B^{\prime} \sigma_{p}^{\prime \prime}\left(x ; R^{+}\right)+C^{\prime} \sigma_{m}^{\prime \prime}\left(x ; R^{-}\right) .
\end{aligned}
$$

The polynomials $R_{1}$ and $R_{2}$ are non-negative over the interval $-1 \leqq x \leqq+1$ and their degrees do not exceed $m$. Clearly (6.1) is satisfied, since

$$
R_{1}(x)-R_{2}(x)=T^{*}(x)-T_{*}(x)=R(x) .
$$

Moreover, using Minkowski's inequality, and taking into account (2.7), we get 
with

$$
\begin{aligned}
\left\{\int_{-1}^{+1} R_{1}^{r} d x\right\}^{1 / r} \leqq & A^{\prime}\left\{\int_{-1}^{+1} \sigma_{n-2}^{\prime \prime r}\left(x ; R^{+}\right) d x\right\}^{1 / r} \\
& +\left|B^{\prime}\right|\left\{\int_{-1}^{+1} \sigma_{p}^{\prime \prime r}\left(x ; R^{-}\right) d x\right\}^{1 / r} \\
& +C^{\prime}\left\{\int_{-1}^{+1} \sigma_{m}^{\prime \prime r}\left(x ; R^{+}\right) d x\right\}^{1 / r} \\
\leqq & K_{m, n}\left\{\int_{-1}^{+1}|R(x)|^{r} d x\right\}^{1 / r},
\end{aligned}
$$

$$
K_{m, n}=A^{\prime}+\left|B^{\prime}\right|+C^{\prime} .
$$

Similarly we prove (6.2) for $i=2$.

A comparison of this argument with that of section 4 shows that the constant $K_{m, n}$ of Theorem 2 is the same as the $K_{m, n}$ of Theorem 1. This immediately gives the inequality (6.2) (cf. (5.1)).

7. It is clear that an argument similar to that of the preceding section (and even slightly simpler, because we may use the first arithmetic means instead of the second) gives an analogue of Theorem 2 for trigonometric polynomials

$$
U(\theta)=(1 / 2) a_{0}+\sum_{\nu=1}^{n}\left(a_{\nu} \cos \nu \theta+b_{\nu} \sin \nu \theta\right) .
$$

TheOREM 3. Let $r \geqq 1$ and let $n \leqq m$ be two positive integers. Every trigonometric polynomial (7.1) of order $n$ may be represented in the form

$$
U(\theta)=U_{1}(\theta)-U_{2}(\theta),
$$

where $U_{1}$ and $U_{2}$ are non-negative trigonometric polynomials of order at most $m$, satisfying the inequalities

$$
\left\{\int_{0}^{2 \pi} U_{i}^{r} d \theta\right\}^{1 / r} \leqq K_{m, n}^{\prime}\left\{\int_{0}^{2 \pi}|U|^{r} d \theta\right\}^{1 / r},
$$

with $K_{m, n}^{\prime}$ depending on $m$ and $n$ only. If $m$ and $n$ satisfy (1.1), then

$$
K_{m, n}^{\prime} \leqq K^{\prime}\left(1+\delta^{-1}\right)
$$

where $K^{\prime}$ is an absolute constant.

The proof may be condensed at some points. Given a function $f(\theta)$ of period $2 \pi$ we shall denote by $s_{n}(\theta ; f)$ and $\sigma_{n}^{\prime}(\theta ; f)$ the partial sums and the first arithmetic means of the Fourier series of $f$. If $p<q$ are two non-negative integers, then the linear combination 


$$
-\frac{n}{m-n+1} \sigma_{n-1}(\theta ; f)+\frac{m+1}{m-n+1} \sigma_{m}(\theta ; f),
$$

written as a trigonometric polynomial, has its $n$th partial sum equal to $s_{n}(\theta ; f)$. Similarly as in section 6 we introduce the functions $U^{+}(\theta)$ and $U^{-}(\theta)$ and the polynomials

$$
\begin{aligned}
& U_{1}(\theta)=\frac{n}{m-n+1} \sigma_{n-1}\left(\theta ; U^{-}\right)+\frac{m+1}{m-n+1} \sigma_{m}\left(\theta ; U^{+}\right), \\
& U_{2}(\theta)=\frac{n}{m-n+1} \sigma_{n-1}\left(\theta ; U^{+}\right)+\frac{m+1}{m-n+1} \sigma_{m}\left(\theta ; U^{-}\right) .
\end{aligned}
$$

These polynomials are non-negative, of degree at most $m$, and satisfy (7.2). Taking in to account that, for any function $f(\theta)$,

$$
\left\{\int_{0}^{2 \pi}\left|\sigma_{\mu}(\theta ; f)\right| r d \theta\right\}^{1 / r} \leqq\left\{\int_{0}^{2 \pi}|f(\theta)| r d \theta\right\}^{1 / r}, \quad 1 \leqq r \leqq+\infty,
$$

we get (7.3) where

$$
K_{m, n}^{\prime}=\frac{m+n-1}{m-n+1}<\frac{2 m+1}{m-n+1} .
$$

This yields (7.4), with $K^{\prime}=2$, provided that $m$ and $n$ satisfy (1.1)

$\S 3$

8. In the proof of Theorem 1, instead of the second arithmetic means $\sigma_{n}^{\prime \prime}(x ; f)$ of the Fourier-Legendre series of the function $f$, we could have used the first arithmetic means $\sigma_{n}^{\prime}(x ; f)$ of that series. The argument would have been similar, for although the first arithmetic means $K_{n}^{\prime}(x, t)$ of the series (2.3) are not positive, they have the property that the integral

$$
\int_{-1}^{+1}\left|K_{n}^{\prime}(x, t)\right| d t
$$

is bounded in $n$ and $x\left({ }^{7}\right)$, and that is the only property of the kernel that was required in the proof. The advantage of using the second arithmetic means is that the positiveness of the kernel $K_{n}^{\prime \prime \prime}(x, t)$ is more elementary than the boundedness of the integral (8.1). Moreover, for the proof of Theorem 2 the fact that we used a positive kernel was essential. On the other hand, using the kernel $K_{n}^{\prime}(x, t)$ we might obtain a better estimate for $K_{m, n}$ than the inequality (5.1). Namely, we might obtain that $K_{m, n}=O\left(\delta^{-1}\right)$ for $\delta \rightarrow+0$. That this estimate is still too crude is shown by the following:

(7) See T. H. Gronwall, Über die Laplacesche Reihe, Math. Ann. vol. 74 (1913) pp. 213-270. 
Theorem 4. Suppose that $0<\delta \leqq 1$, and that $A_{\delta}$ is the least number satisfying (1.10). Then

$$
A_{\delta} \leqq M \delta^{-1 / 2},
$$

where $M$ denotes an absolute constant.

It may be observed that the order $\delta^{-1 / 2}$ in (8.2) cannot be improved. For if, for a given $n$, we take the least possible value of $m$, namely $m=n+1$, then $\delta=1 / n$. On the other hand, the polynomial $P(x)$ is then determined uniquely by its values at the points (1.6). It is, however, well known that a polynomial $P(x)$ of degree $n$, which is absolutely less than 1 at the points (1.6) where $m=n+1$, may be of the order $n^{1 / 2}$ at some points of the interval $-1 \leqq x \leqq+1\left(^{8}\right)$. Hence $\delta^{-1 / 2}$ is the right order of the coefficient $A_{\delta}$.

In the proof of Theorem 3 it is slightly more preferable to use the kernel $K_{n}^{\prime}(x, t)$ instead of $K_{n}^{\prime \prime}(x, t)$. However we shall not appeal to the fact that the integral (8.1) is bounded (on the contrary, our argument would easily prove that fact).

We shall confine our attention to the inequality (1.10). A practically identical argument gives the inequality (1.9), which also may be directly deduced from (1.10) by making $r$ tend to $+\infty$.

Let us revert to the proof of Theorem 1 (see section 4). We start with the inequalities (4.1) and (4.2), where $f$ denotes a polynomial. In the formula (4.3) we replace $T(x)$ by the polynomial

$$
\tau(x)=-\frac{n}{m-n+1} \sigma_{n-1}^{\prime}(x ; f)+\frac{m+1}{m-n+1} \sigma_{m}^{\prime}(x ; f)
$$

which is of degree at most $m$, and which has the property that

Instead of (4.5) we get

$$
s_{n}(x ; \tau)=s_{n}(x ; f) \text {. }
$$

$$
\int_{-1}^{+1} Q(x) \tau(x) d x \leqq\left\{\sum_{\nu=1}^{m}\left|Q\left(x_{\nu}^{(m)}\right)\right|^{r} \gamma_{\nu}^{(m)}\right\}^{1 / r}\left\{\sum_{\nu=1}^{m}\left|\tau\left(x_{\nu}^{(m)}\right)\right|^{r^{\prime}} \gamma_{\nu}^{(m)}\right\}^{1 / r^{\prime}},
$$

where, with the notation of section 4 ,

$$
\left\{\sum_{\nu=1}^{m}\left|\tau\left(x_{\nu}^{(m)}\right)\right|^{r^{\prime}} \gamma_{\nu}^{(m)}\right\}^{1 / r^{\prime}} \leqq L^{\prime}\left\{\int_{-1}^{+1}|\tau(x)|^{r^{\prime}} d x\right\}^{1 / r^{\prime}} .
$$

Theorem 4 will have been established, therefore, when we have shown that

$$
\left\{\int_{-1}^{+1}|\tau(x)| r^{\prime} d x\right\}^{1 / r^{\prime}} \leqq M^{\prime} \delta^{-1 / 2}
$$

where $M^{\prime}$ denotes an absolute constant.

(8) See, for example, G. Szegö, loc. cit. pp. 328-332. 
9. We observe that

$$
\tau(x)=\int_{-1}^{+1} f(t) H(x, t) d t
$$

where

$$
H(x, t)=-\frac{n}{m-n+1} K_{n-1}^{\prime}(x, t)+\frac{m+1}{m-n+1} K_{m}^{\prime}(x, t) .
$$

We shall find for the in tegral

$$
\int_{-1}^{+1}|H(x, t)| d t
$$

a majorant independent of $x$. For this purpose let us consider the first arithmetic means of the series

$$
\sum_{\nu=0}^{\infty}(\nu+1 / 2) P_{\nu}(\cos \gamma)
$$

These means will be denoted by $\chi_{n}(\gamma)$. Using the familiar formula

$$
P_{\nu}(\cos \theta) P_{\nu}\left(\cos \theta^{\prime}\right)=\int_{0}^{2 \pi} P_{\nu}(\cos \gamma) d \phi,
$$

where $\cos \gamma=\cos \theta \cos \theta^{\prime}+\sin \theta \sin \theta^{\prime} \cos \phi$, and setting

$$
\begin{gathered}
x=\cos \theta, \quad t=\cos \theta^{\prime}, \\
h(\gamma)=-\frac{n}{m-n+1} \chi_{n-1}(\gamma)+\frac{m+1}{m-n+1} \chi_{m}(\gamma),
\end{gathered}
$$

we find that

(9.6) $\int_{-1}^{+1}|H(x, t)| d t=\int_{0}^{\pi}\left|\int_{0}^{2 \pi} h(\gamma) d \phi\right| \sin \theta^{\prime} d \theta^{\prime} \leqq \iint_{S}|h(\gamma)| d \omega$

where $S$ denotes the unit sphere and $d \omega$ is the element of area on $S$. It is clear that the last integral, which we shall denote by $I$, is independent of $\theta$, so that we may take for example $\theta=0$. Thus $\theta^{\prime}=\gamma$ and

$$
I=2 \pi \int_{0}^{2 \pi}|h(\gamma)| \sin \gamma d \gamma
$$

Let us now revert to the formula (9.1). Hölder's inequality gives 
so that

$$
\begin{aligned}
|\tau(x)| & \leqq\left\{\int_{-1}^{+1}|f(t)|^{r^{\prime}}|H(x, t)| d t\right\}^{1 / r^{\prime}}\left\{\int_{-1}^{+1}|H(x, t)| d t\right\}^{1 / r} \\
& \leqq I^{\left(r^{\prime}-1\right) / r^{\prime}}\left\{\int_{-1}^{+1}|f(t)| r^{\prime}|H(x, t)| d t\right\}^{1 / r^{\prime}},
\end{aligned}
$$

$$
\begin{aligned}
\int_{-1}^{+1}|\tau(x)| r^{\prime} d x & \leqq I^{r^{\prime}-1} \int_{-1}^{+1} d x \int_{-1}^{+1}|f(t)| r^{\prime}|H(x, t)| d t \\
& =I^{r^{\prime}-1} \int_{-1}^{+1}|f(t)|^{r^{\prime}} d t \int_{-1}^{+1}|H(x, t)| d t \leqq I^{r^{\prime}},
\end{aligned}
$$

on account of (9.6). This fact and (8.6) show that Theorem 4 will be a consequence of the inequality

$$
I \leqq M^{\prime} \delta^{-1 / 2}
$$

which we are now going to prove.

10. We shall use the fact, first pointed out by Fejerr $\left({ }^{9}\right)$, that the series (9.4) is a Cauchy product of the two series

$$
\sum_{\nu=0}^{\infty} P_{\nu}(\cos \gamma), \quad 1 / 2+\sum_{\nu=1}^{\infty} \cos \nu \gamma
$$

Taking into account the familiar formula of Mehler (10)

$$
P_{\mu}(\cos \gamma)=\frac{2}{\pi} \int_{\gamma}^{\pi} \frac{\sin (\mu+1 / 2) t}{[2(\cos \gamma-\cos t)]^{1 / 2}} d t,
$$

we see that the $\nu$ th partial sum of the first series (10.1) is equal to

$$
\frac{2}{\pi} \int_{\gamma}^{\pi} \frac{1-\cos (\nu+1) t}{2 \sin (1 / 2) t[2(\cos \gamma-\cos t)]^{1 / 2}} d t=V_{\nu},
$$

say. Since the $\nu$ th partial sum of the second series (10.1) is the Dirichlet kernel $D_{\nu}(\gamma)=\sin (\nu+1 / 2) \gamma / 2$ sin $(1 / 2) \gamma$, the first arithmetic means $\chi_{N}(\gamma)$ of the series (9.4) are given by the formula

$$
\begin{aligned}
(N+1) \chi_{N}(\gamma) & =\sum_{\nu=0}^{N} V_{\nu}(\gamma) D_{N-\nu}(\gamma) \\
= & \frac{2}{\pi} \cdot \frac{1}{2 \sin \gamma / 2} \int_{\gamma}^{\pi} \frac{\sum_{\nu=0}^{N} \sin (N-\nu+1 / 2) \gamma[1-\cos (\nu+1) t]}{2 \sin (t / 2)[2(\cos \gamma-\cos t)]^{1 / 2}} d t .
\end{aligned}
$$

(9) Fejér, loc. cit.

(10) See, for example, G. Szegö, loc. cit. p. 87. 
Let us now represent here the products of sines by cosines as differences of sines, and let us apply the formulas for the sums of sines of an arithmetic progression. The last formula may then be written

$$
(N+1) \chi_{N}(\gamma)=\frac{2}{\pi} \cdot \frac{1}{2 \sin \gamma / 2} \int_{\gamma}^{\pi} \frac{\alpha_{N}^{(1)}(\gamma)+\alpha_{N}^{(2)}(\gamma, t)+\alpha_{N}^{(3)}(\gamma, t)}{2 \sin (t / 2)[2(\cos \gamma-\cos t)]^{1 / 2}} d t
$$

where

$$
\begin{aligned}
\alpha_{N}^{(1)}(\gamma) & =\frac{1-\cos (N+1) \gamma}{2 \sin \gamma / 2} \\
\alpha_{N}^{(2)}(\gamma, t) & =-\frac{1}{2} \cdot \frac{\cos (N+1) t-\cos (N+1) \gamma}{2 \sin (t-\gamma) / 2} \\
\alpha_{N}^{(3)}(\gamma, t) & =\frac{1}{2} \frac{\cos (N+1) t-\cos (N+1) \gamma}{2 \sin (t+\gamma) / 2}
\end{aligned}
$$

Let us now consider the formula (9.5) and let us set

so that

$$
m-(n-1)=\lambda
$$

$$
h(\gamma)=\lambda^{-1}\left[(m+1) \chi_{m}-(m-\lambda+1) \chi_{m-\lambda}\right] .
$$

Hence, if in (10.2) we substitute for $N$ the values $m$ and $m-\lambda$, and subtract the results, we get

where

$$
h(\gamma) \doteq h^{(1)}(\gamma)+h^{(2)}(\gamma)+h^{(3)}(\gamma)
$$

$$
h^{(i)}(\gamma)=\frac{1}{\lambda} \cdot \frac{2}{\pi} \cdot \frac{1}{2 \sin \gamma / 2} \int_{\gamma}^{\pi} \frac{\alpha_{m}^{(i)}-\alpha_{m-\lambda}^{(i)}}{2 \sin (t / 2)[2(\cos \gamma-\cos t)]^{1 / 2}} d t
$$

We shall show that

$$
\int_{0}^{\pi}\left|h^{(i)}(\gamma)\right| \sin \gamma d \gamma \leqq C(m / \lambda)^{1 / 2}
$$

where $C$ is an absolute constant. (In the rest of this paper we shall denote by $C$ absolute constants, not always the same at every occurrence.) Since the integral $I$ (cf. (9.7)) does not exceed the sum of the integrals (10.5) multiplied by $2 \pi$, and since

$$
\lambda>m-n \geqq n \delta \geqq m \delta / 2,
$$

so that $m / \lambda<2 / \delta$, the inequalities (10.5) will imply (9.8). 
11. Let us take for example $i=2$. We note that

$$
\begin{aligned}
&\left|h^{(2)}(\gamma)\right| \sin \gamma \leqq 2(\pi \lambda)^{-1} \cos (\gamma / 2) \\
& \cdot \int_{\gamma}^{\pi}(2 \sin t / 2)^{-1}[2 \sin (t-\gamma) / 2 \cdot 2 \sin (t+\gamma) / 2]^{-1 / 2}\left|\alpha_{m}^{(2)}-\alpha_{m-\lambda}^{(2)}\right| d t,
\end{aligned}
$$

and we break up the integral on the right into three integrals extended respectively over the intervals $(\gamma, \gamma+1 / m),(\gamma+1 / m, \gamma+1 / \lambda),(\gamma+1 / \lambda, \pi) \cdot{ }^{(1)}$ Correspondingly

$$
\left|h^{(2)}(\gamma)\right| \sin \gamma \leqq R_{1}(\gamma)+R_{2}(\gamma)+R_{3}(\gamma),
$$

where

$$
R_{1}=\lambda^{-1} \cos (\gamma / 2) \int_{\gamma}^{\gamma+1 / m}, \quad R_{2}=\lambda^{-1} \cos (\gamma / 2) \int_{\gamma+1 / m}^{\gamma+1 / \lambda},
$$

Observing that

$$
R_{3}=\lambda^{-1} \cos (\gamma / 2) \int_{\gamma+1 / \lambda}^{\pi} \text {. }
$$

$$
\begin{aligned}
\sin u & \geqq(2 / \pi) u & (0 \leqq u \leqq \pi / 2), \\
\sin (\gamma+t) / 2 & \geqq \cos (\gamma / 2) \sin (t / 2) & (0 \leqq \gamma \leqq t \leqq \pi),
\end{aligned}
$$

we find that

$$
R_{3}(\gamma) \leqq C \lambda^{-1} \int_{\gamma+1 / \lambda}^{\pi} t^{-3 / 2}(t-\gamma)^{-3 / 2} d t \leqq C \lambda^{1 / 2} \int_{1 / \lambda}^{\infty} t^{-3 / 2} d t=C \lambda .
$$

Again,

$$
\begin{aligned}
R_{3}(\gamma) & \leqq C \lambda^{-1} \int_{\gamma+1 / \lambda}^{\infty} t^{-3 / 2}(t-\gamma)^{-3 / 2} d t \leqq C \lambda^{-1} \gamma^{-3 / 2} \int_{\gamma+1 / \lambda}^{\infty}(t-\gamma)^{-3 / 2} d t \\
& =C \lambda^{-1 / 2} \gamma^{-3 / 2}
\end{aligned}
$$

Thus we got the following two inequalities for $R_{3}$ :

$$
R_{3}(\gamma) \leqq C \lambda, \quad R_{3}(\gamma) \leqq C \lambda^{-1 / 2} \gamma^{-3 / 2} .
$$

Taking into account the structure of the numerator of $\alpha^{(2)}$, we find that

(11.3) $\quad R_{2}(\gamma) \leqq C \lambda^{-1} \int_{\gamma+1 / m}^{\gamma+1 / \lambda}\{|\sin \lambda t / 2|+|\sin \lambda \gamma / 2|\} t^{-3 / 2}(t-\gamma)^{-3 / 2} d t$

If we replace each sine on the right by 1 , we get

(11) If any of these intervals partly projects outside $(0, x)$ we integrate over only the part contained in $(0, \pi)$. 


$$
\begin{aligned}
R_{2}(\gamma) & \leqq C \lambda^{-1} \int_{\gamma+1 / m}^{\gamma+1 / \lambda} t^{-3 / 2}(t-\gamma)^{-3 / 2} d t \leqq C \lambda^{-1} \gamma^{-3 / 2} \int_{\gamma+1 / m}^{\infty}(t-\gamma)^{-3 / 2} d t \\
& \leqq C \lambda^{-1} m^{1 / 2} \gamma^{-3 / 2} .
\end{aligned}
$$

If we replace the sines by the corresponding angles and observe that $\gamma \leqq t$, we get

$$
R_{2}(\gamma) \leqq C \int_{\gamma+1 / m}^{\gamma+1 / \lambda} t^{-1 / 2}(t-\gamma)^{-3 / 2} d t \leqq C m^{1 / 2} \gamma^{-1 / 2}
$$

Thus

$$
R_{2}(\gamma) \leqq C m^{1 / 2} \gamma^{-1 / 2}, \quad R_{2}(\gamma) \leqq C \lambda^{-1} m^{1 / 2} \gamma^{-3 / 2} .
$$

Finally, we observe that

$$
R_{1}(\gamma) \leqq C \lambda^{-1} \int_{\gamma}^{\gamma+1 / m} t^{-3 / 2}(t-\gamma)^{-3 / 2}\left|\alpha_{m}^{(2)}-\alpha_{m-\lambda}^{(2)}\right| d t,
$$

and that the numerator of $\alpha_{m}^{(2)}$ is

$$
\sin (1 / 2)(m+1)(t-\gamma) \sin (1 / 2)(m+1)(t+\gamma),
$$

so that the numerator of $\alpha_{m}^{(2)}-\alpha_{m-\lambda}^{(2)}$ is

$$
\begin{aligned}
{[\sin (1 / 2)(m+1)(t-\gamma)-\sin (1 / 2)(m-\lambda+1)(t-\gamma)] \sin (1 / 2)(m+1)(t+\gamma) } \\
+[\sin (1 / 2)(m+1)(t+\gamma)-\sin (1 / 2)(m-\lambda+1)(t+\gamma)] \\
\quad \cdot \sin (1 / 2)(m-\lambda+1)(t-\gamma) \\
=2 \sin (1 / 4) \lambda(t-\gamma) \cos (1 / 2)(m+1-\lambda / 2)(t-\gamma) \\
\quad \cdot \sin (1 / 2)(m+1)(t+\gamma) \\
+2 \sin (1 / 4) \lambda(t+\gamma) \cos (1 / 2)(m+1-\lambda / 2)(t+\gamma) \\
\quad \cdot \sin (1 / 2)(m-\lambda+1)(t-\gamma)
\end{aligned}
$$

If we replace each sine on the right by the corresponding angle, the cosines by 1 , and if we note that $\gamma+t \leqq 2 t$, we find that

$$
\begin{aligned}
R_{1}(\gamma) & \leqq C m \int_{\gamma}^{\gamma+1 / m} t^{-1 / 2}(t-\gamma)^{-1 / 2} d t \leqq C \gamma^{-1 / 2} m \int_{\gamma}^{\gamma+1 / m}(t-\gamma)^{-1 / 2} d t \\
& \leqq C m^{1 / 2} \gamma^{-1 / 2} .
\end{aligned}
$$

However, we may also note that the right-hand side of (11.6) does not exceed

$$
C[\lambda(t-\gamma)+m(t-\gamma)] \leqq C m(t-\gamma)
$$

so that 
Thus

$$
R_{1}(\gamma) \leqq C m \lambda^{-1} \int_{\gamma}^{\gamma+1 / m} t^{-3 / 2}(t-\gamma)^{-1 / 2} d t \leqq C m^{1 / 2} \lambda^{-1} \gamma^{-3 / 2} .
$$

$$
R_{1}(\gamma) \leqq C m^{1 / 2} \gamma^{-1 / 2}, \quad R_{1}(\gamma) \leqq C m^{1 / 2} \lambda^{-1} \gamma^{-3 / 2} .
$$

The inequalities we have proved show that

$$
\int_{0}^{\pi} R_{k}(\gamma) d \gamma \leqq C(m / \lambda)^{1 / 2} \quad(k=1,2,3) .
$$

For $k=3$, this follows immediately if we break up the integral (11.8) into two, extended over the intervals $0 \leqq \gamma \leqq 1 / \lambda$ and $1 / \lambda \leqq \gamma \leqq \pi$, and apply respectively the inequalities (11.2):

$$
\int_{0}^{\pi} R_{3} d \gamma \leqq \int_{0}^{1 / \lambda} C \lambda d \gamma+\int_{1 / \lambda}^{\infty} C \lambda^{-1 / 2} \gamma^{-3 / 2} d \gamma \leqq C \leqq C(m / \lambda)^{1 / 2} .
$$

Similarly, using the inequalities (11.4) and (11.7), we settle the cases $k=2$, 3 of (11.8). Taking into account the definition of the functions $R_{k}$ we deduce from (11.8) the validity of (10.5) for $i=2$.

The case $i=3$ is similar to that of $i=2$, the only difference being that the factor $2 \sin (t-\gamma) / 2$ in the denominator of $\alpha^{(2)}$ is replaced by $2 \sin (t+\gamma) / 2$, which is larger, on account of the obvious inequality

$$
(t-\gamma) / 2 \leqq(t+\gamma) / 2 \leqq \pi-(t-\gamma) / 2 .
$$

Thus the integral (10.5) when $i=3$ is less than the integral with $i=2$.

The case $i=1$ is also easier than that of $i=2$ but is slightly different. We observe that

$$
\begin{aligned}
\cos (\gamma / 2) & \int_{\gamma}^{\pi}(2 \sin t / 2)^{-1}[2(\cos \gamma-\cos t)]^{-1 / 2} d t \\
& \leqq C\left(\int_{\gamma}^{\gamma+1 / \lambda}+\int_{\gamma+1 / \lambda}^{\infty}\right) t^{-3 / 2}(t-\gamma)^{-1 / 2} d t \\
& \leqq C\left[\gamma^{-3 / 2} \int_{\gamma}^{\gamma+1 / \lambda}(t-\gamma)^{-1 / 2} d t+\lambda^{1 / 2} \int_{\gamma+1 / \lambda}^{\infty} t^{-3 / 2} d t\right] \\
& =C\left[\lambda^{-1 / 2} \gamma^{-3 / 2}+\lambda^{1 / 2} \gamma^{-1 / 2}\right] .
\end{aligned}
$$

Of the two terms in the last square brackets, the first prevails for $\gamma \leqq 1 / \lambda$, the second for $\gamma \geqq 1 / \lambda$. Hence, if we denote the left-hand side of (11.9) by $E(\gamma)$, we have the following inequalities

$$
E(\gamma) \leqq C \lambda^{-1 / 2} \gamma^{-3 / 2}, \text { if } \lambda \gamma \leqq 1 ; \quad E(\gamma) \leqq C \lambda^{1 / 2} \gamma^{-1 / 2}, \text { if } \lambda \gamma \geqq 1 .
$$

Obviously 


$$
\begin{aligned}
\left|h^{(1)}(\gamma)\right| \sin \gamma & \leqq C \lambda^{-1} E(\gamma)|\cos (m+1) \gamma-\cos (m-\lambda+1) \gamma| / 2 \sin \gamma / 2 \\
& \leqq C \lambda^{-1} E(\gamma)|\sin (\lambda \gamma / 2) \sin (m+1-\lambda / 2) \gamma| / 2 \sin \gamma / 2 .
\end{aligned}
$$

The right-hand side here may only be increased if the product of sines in the denominator is replaced by one of the following numbers: $\lambda(m+1-\lambda / 2) \gamma^{2} / 2$, $\lambda \gamma / 2,1$. The first will be the most advantageous in the interval $0 \leqq \gamma \leqq 1 / m$, the second for $1 / m \leqq \gamma \leqq 1 / \lambda$, the third for $\gamma \geqq 1 / \lambda$. Taking into account (11.10), we deduce from (11.11) that $\left|h^{(1)}(\gamma)\right| \sin \gamma$ is majorized by the following expressions

$$
C m \lambda^{-1 / 2} \gamma^{-1 / 2}, \quad \text { if } \quad 0 \leqq \gamma \leqq 1 / m ; \quad C \lambda^{-1 / 2} \gamma^{-3 / 2}, \quad \text { if } \quad \gamma \geqq 1 / m,
$$

and these inequalities give (10.5) for $i=1$. This completes the proof of Theorem 4.

12. It may be of some interest to point out that the constants $A_{\delta}^{\prime}$ and $B_{\delta}^{\prime}$, of the inequalities (1.3) and (1.4) are $O(\log 1 / \delta)$ as $\delta \rightarrow+0$. For the inequality (1.3) this is proved in Bernstein's paper cited above. The inequality for $B_{\delta}^{\prime}$ follows almost immediately from the argument used in the paper by Marcinkiewicz and Zygmund( $\left.{ }^{(}\right)$, provided we use the fact that, if

$$
K_{n}(t)=(n+1)^{-1}\left\{\sin ^{2}(n+1) t / 2\right\} / 2 \sin ^{2} t / 2
$$

denotes Fejér's kernel, and $0<\lambda<m$, then

$$
\int_{0}^{\pi} \lambda^{-1}\left|(m+1) K_{m-1}(t)-(m-\lambda+1) K_{m-\lambda}(t)\right| d t=O(1+\log m / \lambda) .
$$

For the proof of this inequality it is sufficient to split up this integral into three, extended respectively over the in tervals $(0,1 / m),(1 / m, 1 / \lambda),(1 / \lambda, \pi)$, and to observe that in the first integral the integrand is $O(m)$, in the second it is $O(1 / t)$, and in the third $O\left(1 / \lambda t^{2}\right)$.

MT. Holyoke College, South Hadley, Mass. 